\title{
A Novel Ball on Beam Stabilizing Platform with Inertial Sensors
}

\author{
Part I: Modeling \& Simulation with Detailed Geometrical Analysis
}

\author{
Ali Shahbaz Haider \\ Electrical Engineering Dept \\ COMSATS Institute of Information Technology \\ Wah, Pakistan \\ Muhammad Nasir \\ Electrical Engineering Dept. \\ COMSATS Institute of Information Technology \\ Wah, Pakistan
}

\author{
Basit Safir \\ Electrical Engineering Dept. \\ COMSATS Institute of Information Technology \\ Wah, Pakistan \\ Farhan Farooq \\ Electrical Engineering Dept. \\ COMSATS Institute of Information Technology \\ Wah, Pakistan
}

\begin{abstract}
This research paper presents dynamic modeling of inertial sensor based one degree of freedom (1-DoF) stabilizing platform. Plant is a ball on a pivoted beam. Nonlinear modeling of the plant is done. Ball position on beam is actuated by DC motor using two arms and one beam structure. Arms and beam are linked by pivoted joints. Nonlinear geometrical relations for mechanical structure are derived followed by physically realizable approximations. These relations are used in system dynamic equations followed by linearization, resulting in a linear continuous time differential equation model. State space conversion is done. Final model is simulation and system dynamics are elaborated by analysis of the simulation responses
\end{abstract}

Keywords-stabilizing platform; ball on beam; nonlinear dynamics; inertial sensors

\section{INTRODUCTION}

Stabilizing platforms are among challenging control systems. Their applications are immense, especially in defense such as camera stabilizations for drones and automatic gun pointing angle control. Such plate forms have been benchmarks to practice various control techniques. Owing to such significance a lot of research work has been dedicated to these systems. One of such systems is single degree of freedom (1DoF) ball on beam mechanism. Plant of this control problem consists of a ball capable of rolling on a beam under the action of gravity due to inclination of beam. Control objective is to stabilize the positions of the ball on the beam in the presence of external disturbances and to achieve ball position reference tracking. System is open loop unstable so feedback is inevitable [1].

This research is presented in two parts. Part-I presents modeling of the system. Dynamics of this plant has been derived and discussed in literature. Reduced order linear transfer function based model is used in [1], [2] and [4]. Similar technique has been employed in [3]. Variational techniques have been employed in [5]. However full order model without neglecting actuator dynamics and structural nonlinearities is not studied.
Our work presents geometrically accurate, nonlinear and detailed modeling which has not been presented in literature. Moreover concept of using inertial sensors i.e. rate gyro and accelerometer to measure systems states, is novel and it makes our system much closer to real stabilizing platforms in sea ships and aircraft. In this paper a nonlinear system model is developed followed by linearization and state space conversion. Various systems parameters are identified and their effects on systems dynamics are elucidated. Linear and nonlinear relations are compared and error due to linearization has been analyzed. Open loop linearized dynamics are simulated and discussed.

Organization of the paper is as follows, section-II describes the design and construction of physical hardware. Section-III comprises of derivation of system dynamics, nonlinear geometric relations governing the physical hardware, linearization and state-space conversion. Section-IV presents simulation results followed by section- $\mathrm{V}$ describing conclusions and future work.

\section{HARDWARE DESIGN}

Hardware platform is shown in Figure 1. Functional description for this plant is described diagrammatically in Figure 2. Plant consists of a beam of length $2 b$ hinged at its centre at the pivot point $O_{2}$. A ball is placed on this beam. Ball is capable of rolling freely. Its distance $D$ from edge of the beam is to be controlled. Position of the ball changes under the action of gravity if the beam is inclines at some angle $\theta$, which may be positive or negative. Inclination of beam is actuated by a DC gear motor which is connected to the beam by a servo arm and a link arm. DC gear motor is bidirectional and actuates the servo arm at an angle $\phi$. Servo arm rotates about point $O_{1}$, which is also taken to be the origin of rectangular coordinate system used to model the system. Link arm is pivoted at points $P_{2}$ and $P_{1}$, these pivot points track a circular trajectory in response to angle $\phi$ as shown by dashed circular trajectories in the Figure 2. 


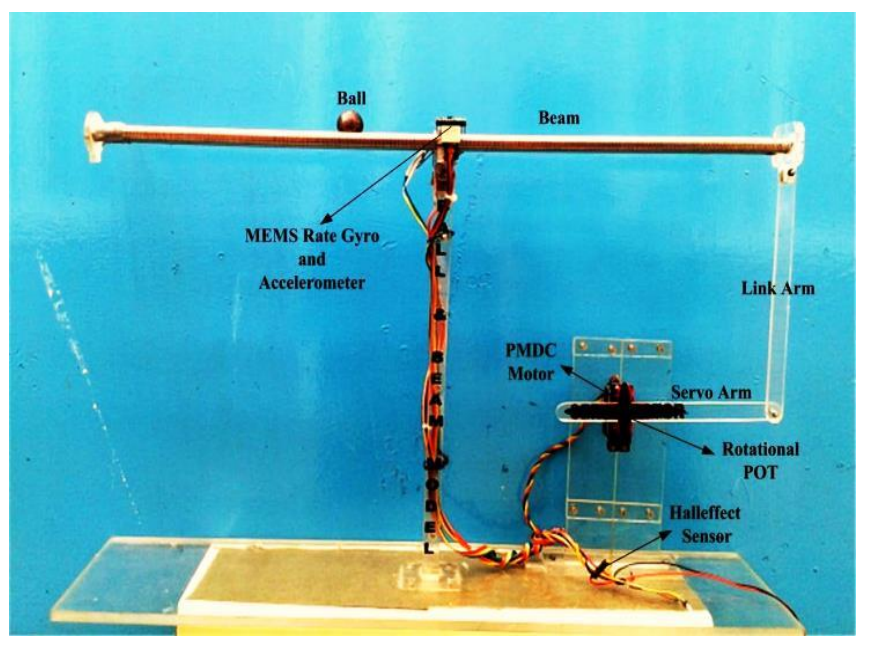

Fig. 1. Hardware platform

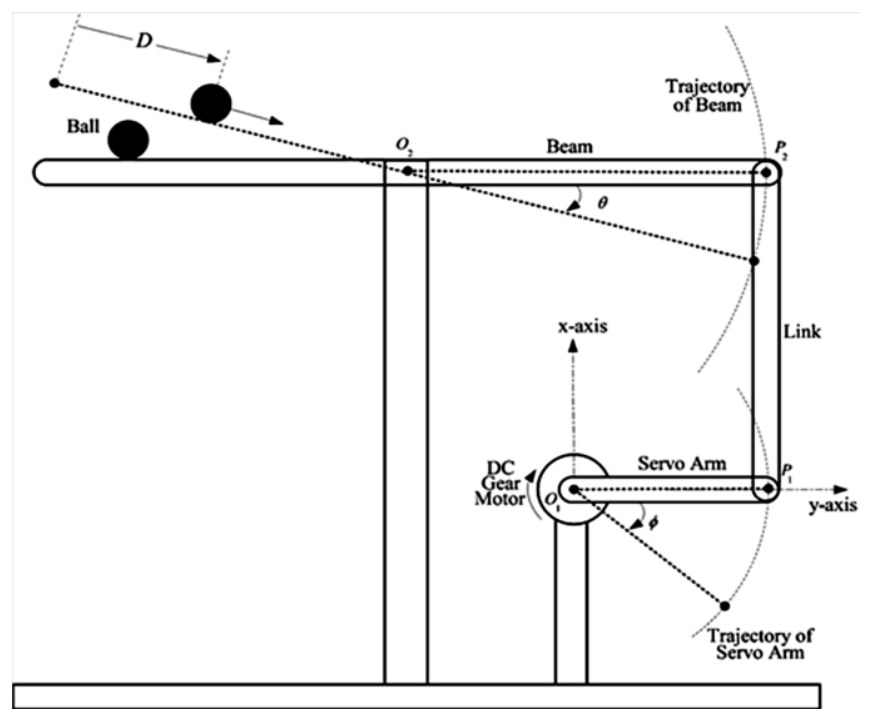

Fig. 2. Functional description of the hardware platform

Control objective is to stabilize the ball at any desired position $D$ or to make it track a commanded position trajectory $D(\mathrm{t})$ by adjusting bounded servo angle $\phi$ in the presence of disturbances.

\section{SYSTEM DYNAMICS}

It can be seen in Figure 2 that trajectories of the servo arm and the beam are circular so mathematical relations will be nonlinear. In the following sub sections various geometrical relations for the system hardware are developed step by step to be used in the final system dynamic model.

\section{A. DC motor dynamics}

The servo arm in Figure 2 is actuated to an angle $\phi$ by a DC gear motor. Model of a permanent magnet (PM) DC motor is shown in Figure 3.

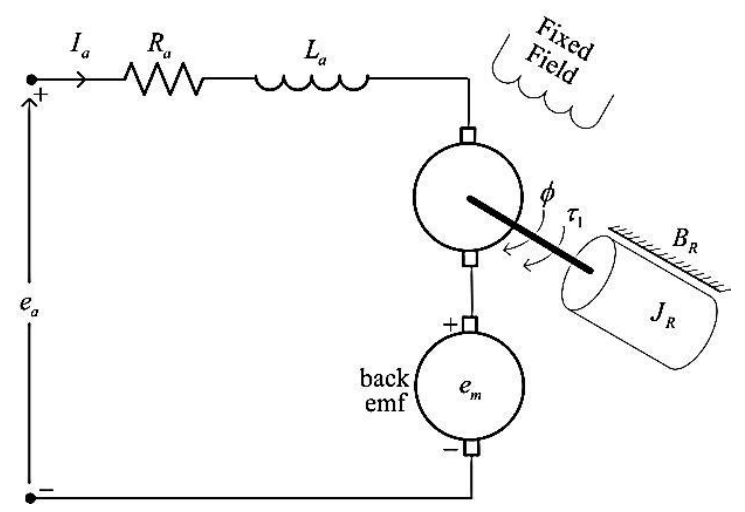

Fig. 3. Electrical and mechanical modeling diagram of PMDC motor

It has two parts namely electrical and mechanical. Armature part of PMDC motor is modelled by a series RL circuit with back emf $e_{m}$ as shown in Figure 3. Input to the motor is voltage signal $e_{a}$.

$$
\begin{gathered}
e_{a}-e_{m}=R_{a} i_{a}+L_{a} \frac{d i_{a}}{d t} \\
e_{m}=k_{m} \frac{d \phi}{d t}
\end{gathered}
$$

Substituting equation (2) in equation (1) we get:

$$
e_{a}-k_{m} \frac{d \phi}{d t}=R_{a} i_{a}+L_{a} \frac{d i_{a}}{d t}
$$

Mechanical part of the motor consists of the rotating armature with its end coupled to the servo arm and the link arm. Armature is modelled by a cylindrical moment of inertia $J_{R}$ experiencing a friction $B_{R}$ as shown in Figure 3. Since motor output shaft is coupled to servo arm and link arm so net moment of inertia is $J_{1}$ given by equation (4),

$$
J_{1}=J_{R}+J_{S}+J_{L}
$$

In Equation (4), $J_{S}$ and $J_{L}$ are moment of inertia of the servo arm and the link arm respectively. Using Euler's equations for mechanical rotation part of motor we obtain,

$$
J_{1} \frac{d^{2} \phi}{d t^{2}}=\tau_{1}-B_{R} \frac{d \phi}{d t}
$$

Electromechanical coupling equation is given by:

$$
\tau_{1}=k_{\tau} i_{a}
$$

\section{B. Expressions for moment of inertia}

Armature is modelled by a cylinder of diameter $r$ and mass $m_{R}$. The Servo arm, link arm and the beam are modelled by rectangular parallelepiped with dimensions shown in Figure 4. Their moment of inertia are given by following equations, 


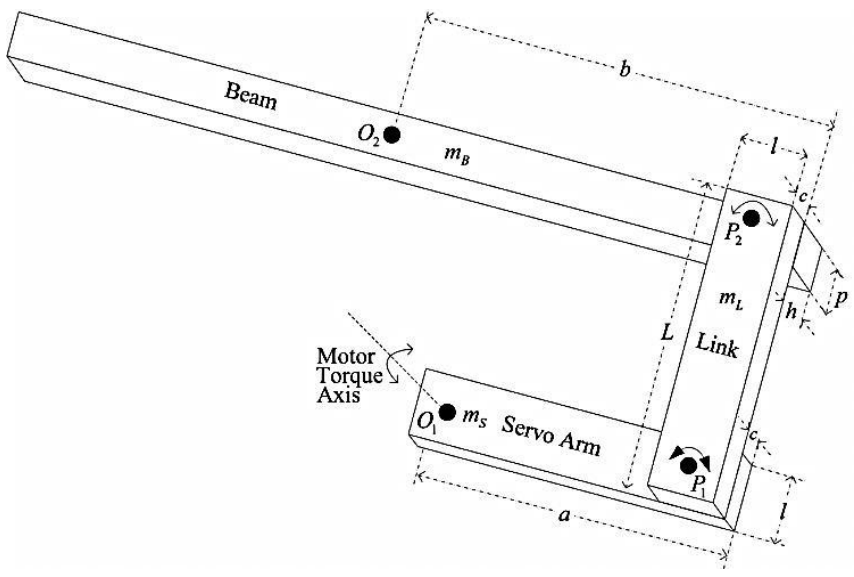

Fig. 4. Moment of inertia for the servo arm, link arm and the beam

$$
\begin{gathered}
J_{R}=\frac{1}{2} m_{R} r^{2} \\
J_{S}=\frac{1}{12} m_{S} l^{2}+\frac{1}{3} m_{S} a^{2} \\
J_{L}=\frac{1}{12} m_{L} l^{2}+\frac{1}{3} m_{L} L^{2}+m_{L} a^{2} \\
J_{2}=\frac{1}{12}\left(m_{B}(2 b)^{2}+m_{S} p^{2}\right)
\end{gathered}
$$

\section{Relation Between servo arm angle $\phi$ and beam angle $\theta$}

Position of the ball is actuated by beam angle $\theta$ as shown in Figure 2. Beam angle is itself result of servo arm angle $\phi$, so a relationship is developed between these two angles. To develop this relation we select a xy-coordinate system with origin $O_{1}(0,0)$ as shown in Figure 5. If $\left|\overline{O_{1} P_{1}}\right|=a$ and $\left|\overline{O_{2} P_{2}}\right|=b$ then beam rotates about $O_{2}(a-b, L)$. Link arm end points become $P_{1}(\mathrm{a} \cos \phi, a \sin \phi)$ and $P_{2}(b \cos \theta-b+a, b \sin \theta+L)$.

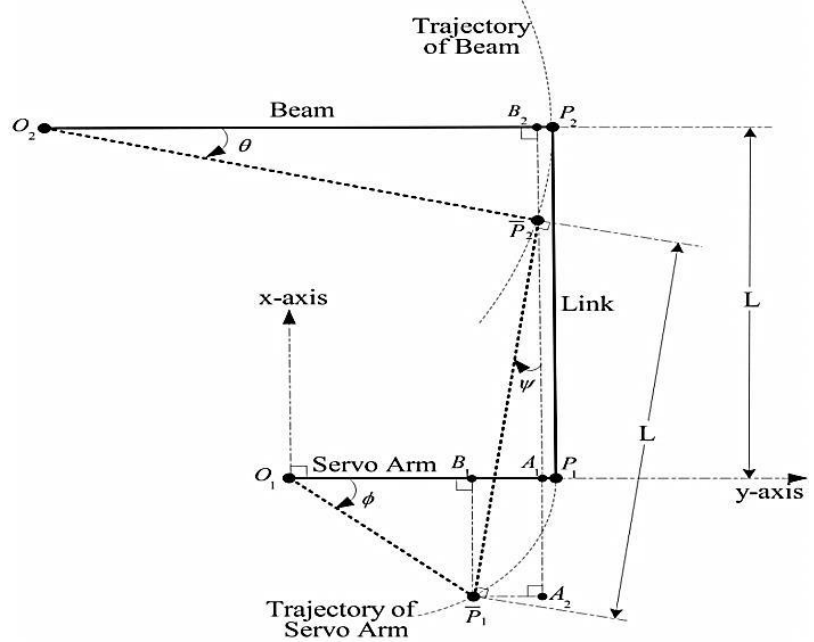

Fig. 5. Geometrical description of the hardware platform
It is clear that $\left|\overline{P_{1} P_{2}}\right|=L$ which remain constant irrespective of position of link arm. We may also write it as $\left|\overline{P_{1} P_{2}}\right|^{2}=L^{2}$ or $\left|\overrightarrow{O_{1} P_{2}}-\overrightarrow{O_{1} P_{1}}\right|^{2}=L^{2}$. From Figure 5 we can expand this relation using distance formula as:

$$
\begin{aligned}
& (b \cos \theta-b+a-\mathrm{a} \cos \phi)^{2}+(b \sin \theta+L-a \sin \phi)^{2}=L^{2} \\
\Rightarrow & (b \cos \theta-b+a-\mathrm{a} \cos \phi)^{2}+(b \sin \theta+L-a \sin \phi)^{2}-L^{2}=0 \\
\Rightarrow & f(a, b, L, \phi, \theta)=0
\end{aligned}
$$

Equation (11) can easily be verified by checking physically realizable trivial solution of $f(a, b, L, 0, \theta)$ or $-b^{2} \cos \theta+b L \sin \theta$ which comes out to be $\theta=0$ as expected. Equation (11) is tedious to be solved to find an explicit relation between $\theta$ and $\phi$. However if we put constrain $\theta \in[-0.1 \pi, 0.1 \pi]$ we may approximate $\sin \theta=\theta$ and $\cos \theta=1$, so equation (11) results in,

$$
\begin{aligned}
& f(a, b, L, \phi, \theta \in[-0.1 \pi, 0.1 \pi])=0 \\
& \Rightarrow {\left[a^{2}\right] \cos \phi+[a b(\theta+1)] \sin \phi=a^{2}+b L \theta } \\
& \Rightarrow \sqrt{\left[a^{2}\right]^{2}+[a b(\theta+1)]^{2}} \times \\
& \cos \left\{\phi+\tan ^{-1}\left[\frac{a b(\theta+1)}{a^{2}}\right]\right\}=a^{2}+b L \theta
\end{aligned}
$$

At this point we can get the following explicit relation between $\theta$ and $\phi$ :

$$
\phi=\cos ^{-1}\left[\frac{1+\frac{b}{a^{2}} L \theta}{\sqrt{1+\left[\frac{b}{a}(\theta+1)\right]^{2}}}\right]+\tan ^{-1}\left[\frac{b}{a}(\theta+1)\right]
$$

Putting $b=2 a$ in equation (13) we get,

$$
\phi=\cos ^{-1}\left[\frac{1+(2 L \theta / a)}{\sqrt{1+4(\theta+1)^{2}}}\right]+\tan ^{-1}[2(\theta+1)]
$$

Furthermore if $L=a$ then Equation (14) becomes:

$$
\phi=\cos ^{-1}\left[\frac{2(\theta+1)-1}{\sqrt{1+[2(\theta+1)]^{2}}}\right]+\tan ^{-1}[2(\theta+1)]
$$

An approximate linear relation can be found between $\theta$ and $\phi$ if we restrain both $\theta$ and $\phi$ in range $[-0.1 \pi, 0.1 \pi]$. In this case Equation (11) becomes:

$$
\begin{aligned}
& f(a, b, L, \phi \in[-0.1 \pi, 0.1 \pi], \theta \in[-0.1 \pi, 0.1 \pi])= \\
& a^{2}+[a b(\theta+1)] \phi-a^{2}-b L \theta=0, \\
& \Rightarrow \theta=\frac{a}{L} \phi .
\end{aligned}
$$


For $\theta=[-0.1 \pi, 0.1 \pi], \%$ error between $\phi$ calculated from nonlinear relation of equation (13) and linear relation of equation (16) have been plotted for various values of $L$ in figure 6. It is evident that linear relation of equation (16) is valid for $\phi \in[-0.1 \pi, 0.1 \pi]$ and $\theta \in[-0.035 \pi, 0.05 \pi]$ if $L=a$, with a maximum of $5 \%$ error.

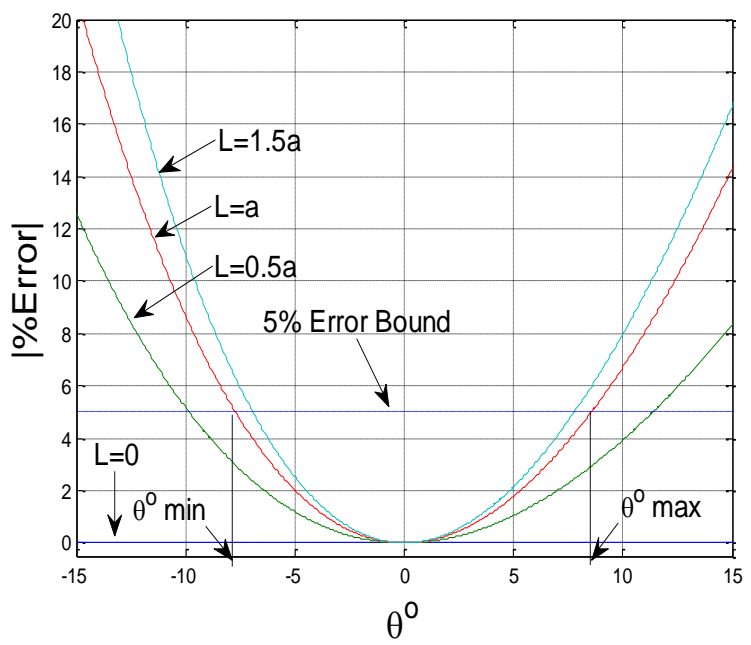

Fig. 6. \% error between linear and nonlinear relation for $\phi$

\section{Torque and force decomposition for the Servo-arm and the link-arm}

PMDC motor generates a torque $\tau_{1}$ and angle $\phi$ which is applied to servo arm at point $O_{1}$ as shown in Figure 7.Since length of the servo arm is 'a' so force $F_{s}$ normal to arm at point $\bar{P}_{1}$ is produces. Amplitude of this force is given by $F_{s}=\tau_{1} / a$. However we are interested in finding the component of force $F_{L}$ which is parallel to the link arm so we decompose $F_{s}$ along $L_{\mathrm{0}}$-axis which comes out to be,

$$
F_{L}=F_{S} \cos \left(\frac{\pi}{2}-\eta\right)
$$

In equation (17) the unknown angle $\eta$ needs to be expressed in terms of known angle $\phi$. Using law of sine in right $\Delta \bar{P}_{1} A_{2} \bar{P}_{2}$ in Figure 5 we get,

$$
\psi=\sin ^{-1} \frac{a(1-\cos \phi)}{L}
$$

In right $\Delta O_{1} B_{1} \bar{P}_{1}$ in Figure 5 we have,

$$
\eta-\psi=\frac{\pi}{2}-\phi
$$

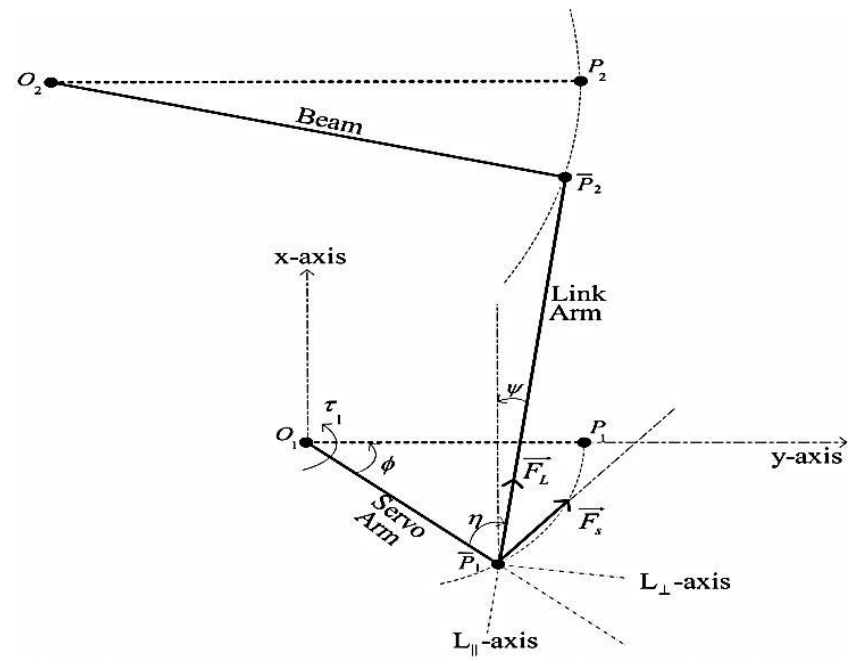

Fig. 7. Electrical and mechanical modeling diagram of PMDC motor

Using Equation (18) in Equation (19) we get:

$$
\eta=\frac{\pi}{2}-\phi+\sin ^{-1} \frac{a(1-\cos \phi)}{L}
$$

Using Equation (20) in Equation (17) we get the desired expression for $F_{L}$ as:

$$
F_{L}=\frac{\tau_{1}}{a} \cos \left(\phi-\sin ^{-1} \frac{a(1-\cos \phi)}{L}\right)
$$

\section{E. Torque and force decomposition for the Link-arm and the beam}

The force $F_{L}$ is transmitted from link arm to the beam. Figure 8 shows that this force is incident on the beam at an angle of $\lambda$ from beam axis. Component of this force $F_{L \perp}$ perpendicular to beam axis is responsible for producing torque about point $O_{2}$. This torque is given by,

$$
\tau_{2}=b F_{L \perp}=b F_{L} \sin (\lambda)
$$

The unknown angle $\lambda$ needs to be expressed in terms of input angle $\phi$. From Figure 8 we have,

$$
\lambda=\frac{\pi}{2}-\psi-\theta
$$

Using equations (16)-(20) in equation (23) we get,

$$
\lambda=\frac{\pi}{2}-\sin ^{-1} \frac{a(1-\cos \phi)}{L}-\frac{a}{L} \phi
$$

Substituting equation (24) in (22) we get,

$$
\tau_{2}=b F_{L} \sin \left(\frac{\pi}{2}-\sin ^{-1} \frac{a(1-\cos \phi)}{L}-\frac{a}{L} \phi\right)
$$




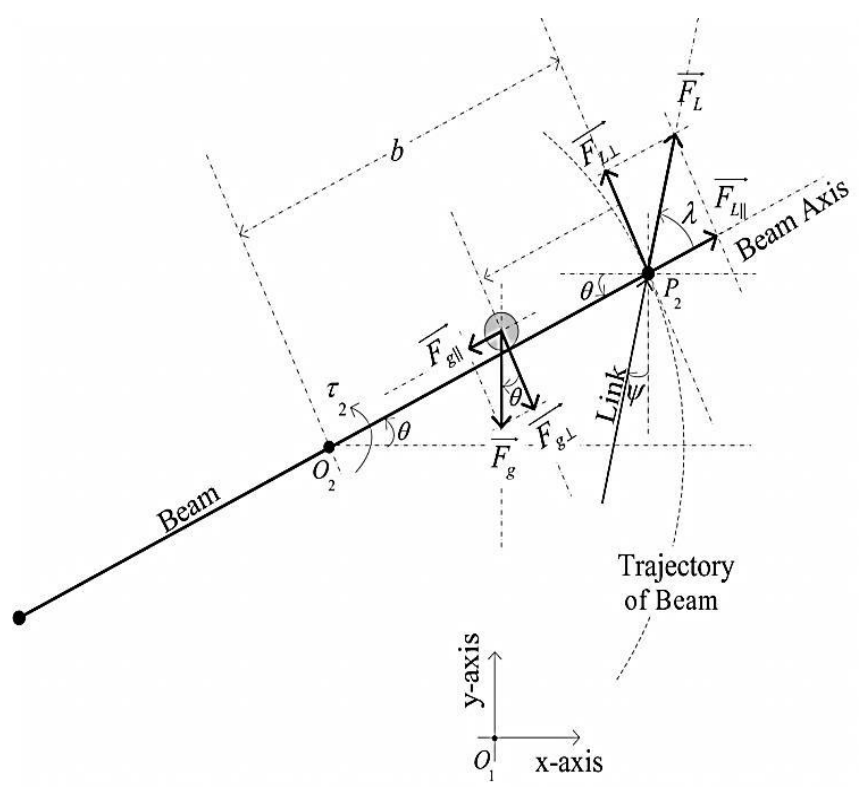

Fig. 8. Torque and force decomposition for the Link-arm and the beam

Using equation (21) in equation (25) we get,

$$
\begin{aligned}
\tau_{2}= & b \frac{\tau_{1}}{a} \cos \left(\phi-\sin ^{-1} \frac{a(1-\cos \phi)}{L}\right) \times \\
& \sin \left(\frac{\pi}{2}-\sin ^{-1} \frac{a(1-\cos \phi)}{L}-\frac{a}{L} \phi\right)
\end{aligned}
$$

It is also worth noting that for small $\phi$ and $a=L$ equation (26) becomes:

$$
\left.\tau_{2}\right|_{\phi \in[-0.1 \pi, 0.1 \pi], a=L}=\frac{b}{a} \tau_{1}
$$

\section{F. Dynamics of motion of the ball and the beam}

Beam experiences a torque $\tau_{2}$ given by equations (26) and (27). as,

Using Euler's Law we get beam dynamics from Figure 8

$$
J_{2} \frac{d^{2} \theta}{d t^{2}}=\tau_{2}-B_{2} \frac{d \theta}{d t}
$$

Dynamics of the ball are given by Newton's Law,

$$
m \frac{d^{2} D}{d t^{2}}=F_{g \square}-F_{f}
$$

Substituting the values of frictional force $F_{f}$ and component of gravitational force parallel to beam $F_{g \square}$ in equation (29) we get,

$$
m \frac{d^{2} D}{d t^{2}}=m g \sin \theta-B \frac{d D}{d t}
$$

For $\theta$ and $\phi$ in range $[-0.1 \pi, 0.1 \pi]$ equation (30) becomes,

$$
m \frac{d^{2} D}{d t^{2}}=m g \theta-B \frac{d D}{d t}
$$

\section{G. State space description of the system}

Four differential equations (32) describe the overall system dynamics. Using Equation (3) and (31) we get:

$$
\begin{aligned}
& \frac{d i_{a}}{d t}=-\frac{R_{a}}{L_{a}} i_{a}-\frac{k_{m}}{L_{a}} \frac{d \phi}{d t}+\frac{1}{L_{a}} e_{a} \\
& \frac{d^{2} D}{d t^{2}}=g \theta-\frac{B}{m} \frac{d D}{d t}
\end{aligned}
$$

Using equations (27) and equation (6) in (28) and (5) we get:

$$
\begin{aligned}
& \frac{d^{2} \phi}{d t^{2}}=\frac{k_{\tau}}{J_{1}} i_{a}-\frac{B_{R}}{J_{1}} \frac{d \phi}{d t} \\
& \frac{d^{2} \theta}{d t^{2}}=\frac{k_{\tau} b}{a J_{2}} i_{a}-\frac{B_{2}}{J_{2}} \frac{d \theta}{d t}
\end{aligned}
$$

Table 1 describes state variable assignment for equation (32 $\alpha, 32 \beta$ ). Using these definitions we get following state space equations $(33 \alpha, 32 \beta)$.

$$
\begin{gathered}
\dot{x}_{1}=\dot{D}=x_{2} \\
\dot{x}_{2}=\ddot{D}=g x_{3}-\frac{B}{m} x_{2} \\
\dot{x}_{3}=\dot{\theta}=x_{4} \\
\dot{x}_{4}=\ddot{\theta}=\frac{k_{\tau} b}{a J_{2}} x_{7}-\frac{B_{2}}{J_{2}} x_{4} \\
\dot{x}_{5}=\dot{\phi}=x_{6} \\
\dot{x}_{6}=\ddot{\phi}=\frac{k_{\tau}}{J_{1}} x_{7}-\frac{B_{R}}{J_{1}} x_{6} \\
\dot{x}_{7}=\dot{i}_{a}=-\frac{R_{a}}{L_{a}} x_{7}-\frac{k_{m}}{L_{a}} x_{6}+\frac{1}{L_{a}} e_{a}
\end{gathered}
$$

Although we are primarily interested in stabilizing the position of the ball $x_{1}$ but to get a good control of stabilizing platform i.e. beam in this case, others state variables are also measures and designated as outputs. From table 1 we have defined following output variables,

$$
\begin{aligned}
& y_{1}=k_{D} x_{1} \\
& y_{2}=k_{\theta} x_{3} \\
& y_{3}=k_{\phi} x_{5} \\
& y_{4}=k_{i} x_{7}
\end{aligned}
$$

The state space equations (33)-(34) can be put into matrix form resulting in the state space quadruple given by, 


$$
A=\left[\begin{array}{ccccccc}
0 & 1 & 0 & 0 & 0 & 0 & 0 \\
0 & -B / m & g & 0 & 0 & 0 & 0 \\
0 & 0 & 0 & 1 & 0 & 0 & 0 \\
0 & 0 & 0 & -\mathrm{B}_{2} / \mathrm{J}_{2} & 0 & 0 & 2 k_{\tau} / J_{2} \\
0 & 0 & 0 & 0 & 0 & 1 & 0 \\
0 & 0 & 0 & 0 & 0 & -B_{R} / J_{1} & k_{\tau} / J_{1} \\
0 & 0 & 0 & 0 & 0 & -k_{m} / L_{a} & -R_{a} / L_{a}
\end{array}\right],
$$

$B=\left[\begin{array}{lllllll}0 & 0 & 0 & 0 & 0 & 0 & 1\end{array}\right]^{T}$,

$$
C=\left[\begin{array}{ccccccc}
k_{D} & 0 & 0 & 0 & 0 & 0 & 0 \\
0 & 0 & k_{\theta} & 0 & 0 & 0 & 0 \\
0 & 0 & 0 & k_{d \theta / d t} & 0 & 0 & 0 \\
0 & 0 & 0 & 0 & k_{\phi} & 0 & 0 \\
0 & 0 & 0 & 0 & 0 & 0 & k_{i}
\end{array}\right],
$$

$$
D=\left[\begin{array}{lllll}
0 & 0 & 0 & 0 & 0
\end{array}\right]^{T}
$$

Using table 2 for values of various parameters of the system in equation (35) we obtain following final state space model of equation (36).

\section{Simulation Results}

System described by equation (35) is a single input multiple output (SIMO) system. Response of the system to unit step input voltage is shown in figure 9-13. It is evident that ball's position $D$ in figure 9 is unstable.

$$
\begin{aligned}
A & =\left[\begin{array}{ccccccc}
0 & 1 & 0 & 0 & 0 & 0 & 0 \\
0 & -0.82 & 9.8 & 0 & 0 & 0 & 0 \\
0 & 0 & 0 & 1 & 0 & 0 & 0 \\
0 & 0 & 0 & -341.3 \mathrm{e} 3 & 0 & 0 & 54.6 e 3 \\
0 & 0 & 0 & 0 & 0 & 1 & 0 \\
0 & 0 & 0 & 0 & 0 & -13.65 & 1.36 \\
0 & 0 & 0 & 0 & 0 & -61.2 e-3 & -3.26
\end{array}\right] \\
C & =\left[\begin{array}{ccccccc}
5 & 0 & 0 & 0 & 0 & 0 & 0 \\
0 & 0 & 4 & 0 & 0 & 0 & 0 \\
0 & 0 & 0 & 2.5 & 0 & 0 & 0 \\
0 & 0 & 0 & 0 & 3.5 & 0 & 0 \\
0 & 0 & 0 & 0 & 0 & 0 & 4.5
\end{array}\right]
\end{aligned}
$$

Instability in the position of the ball is due to the fact that ball accelerates under the effect of gravity on inclined beam so its velocity increases linearly and distance covered increases in a quadratic fashion. In figure 10 and 12 , the beam angle $\theta$ and servo arm angle $\phi$ keep on increasing as motor starts rotating however as motor current stabilizes due to back emf so does the velocity of the motor. This is evident from figure 11 and 13. Hence responses of $D, \theta$ and $\phi$ are bounded input bounded output (BIBO) unstable while armature current $i_{a}$ and beams angular velocity $d \theta / d t$ are BIBO stable.

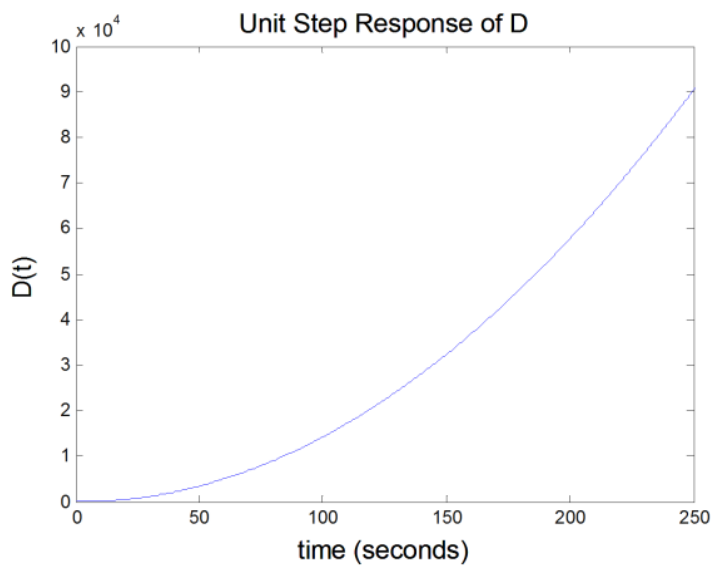

Fig. 9. Unit step response of ball position D

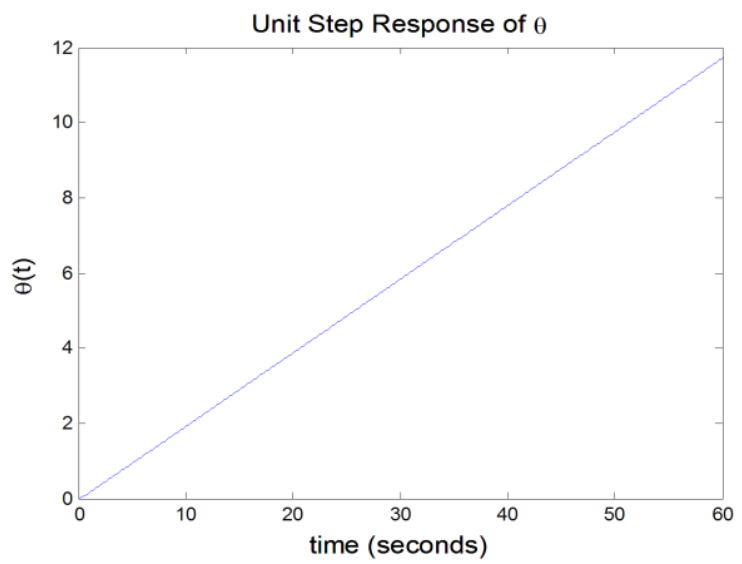

Fig. 10. Unit step response of beam angle $\theta$

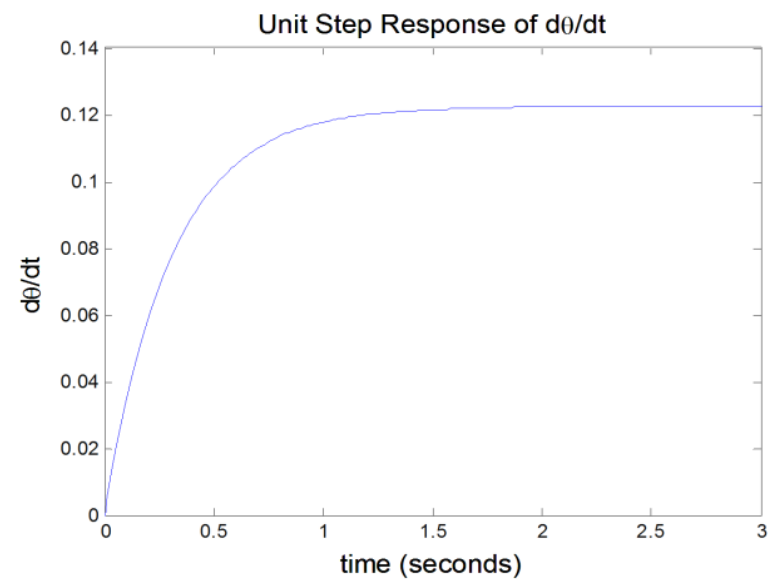

Fig. 11. Unit step response of beam angular velocity $\mathrm{d} \theta / \mathrm{dt}$ 


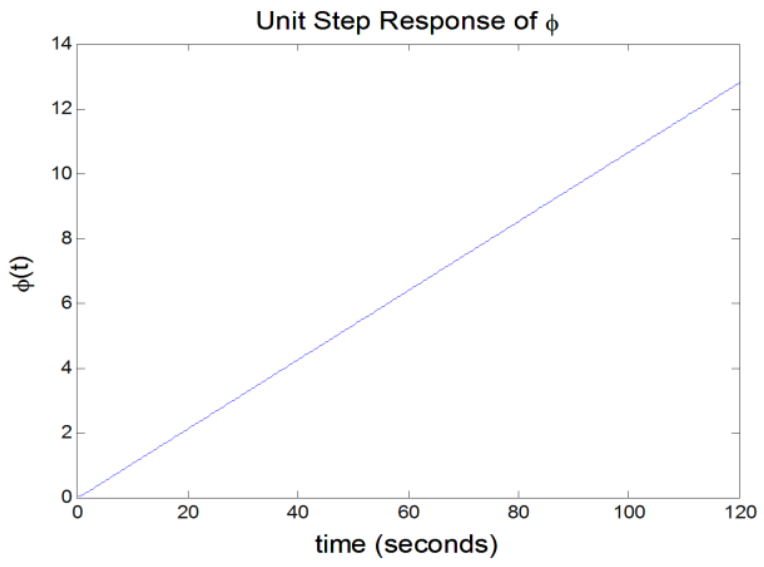

Fig. 12. Unit step response of servo arm angle $\phi$

\section{CONCLUSIONS AND FUTURE WORK}

The detailed geometric analysis of the system shows that equations governing the system behavior are highly non-linear and relations are mostly implicit. Linearization of system dynamic is valid in very narrow operating range. Analysis of the system equations and response indicate that the system has overall unstable response and it is challenging to be controlled. Feedback is inevitable owing to the unstable nature of the system and model inaccuracies caused by linearization of dynamic. In part-II of this research work control of this system would be developed.

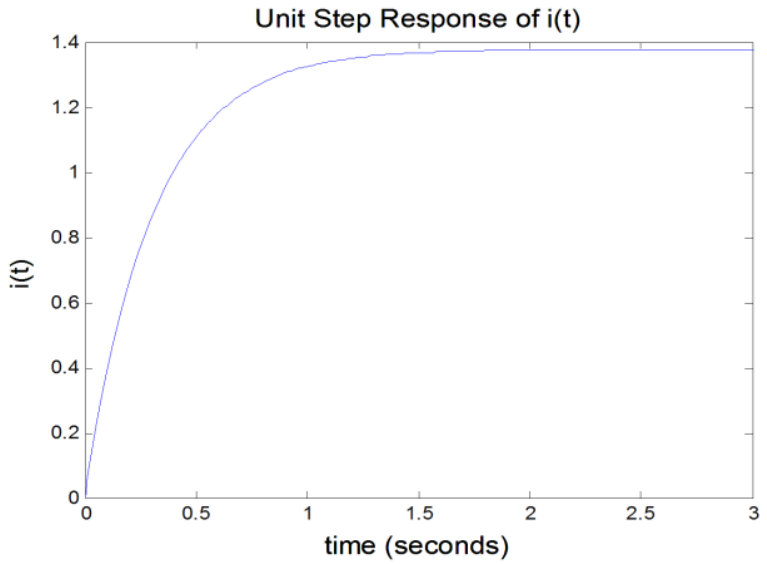

Fig. 13. Unit step response of armature current $i_{a}(t)$

\section{REFERENCES}

[1] K. Ogata. Modern Control Engineering. 3rd ed., New Jersey: Prentice Hall, 1997.

[2] P. R. Bélanger, Control Engineering: A Modern Approach, USA: Saunders College Pub., 1995.

[3] M. Keshmiri, A.F. Jahromi, A. Mohebbi, M. H. Amoozgar, and W. F. Xie. "Modeling and control of ball and beam system using model based and non-model based control approaches." International Journal on smart sensing and intelligent systems 5, vol. 1, pp. 14-35, 2012

[4] Y. Wen, and F. Ortiz. "Stability analysis of PD regulation for ball and beam system." In Control Applications, 2005. CCA 2005. Proceedings of 2005 IEEE Conference on, pp. 517-522., 2005.

[5] P. E. Wellstead., V. Chrimes, P. R. Fletcher, R. Moody, and A. J. Robins. "The ball and beam control experiment." International Journal of Electrical Engineering Education 15, vol. 1, pp. 21-39. 1978

TABLE I. STATE VARIABLE ASSIGNMENT

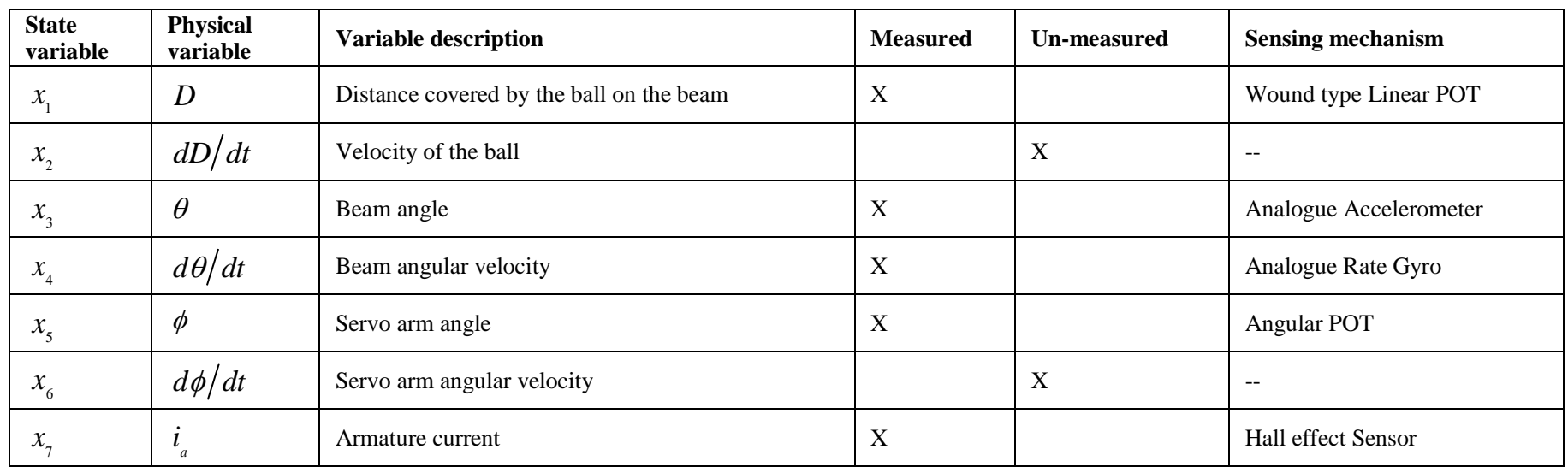


TABLE II. VALUES OF THE SYSTEM PARAMETERS

\begin{tabular}{|c|c|c|}
\hline Parameter Description & Symbol & Numerical Value \\
\hline Acceleration due to Gravity & $g$ & $9.8 \mathrm{~m} / \mathrm{sec}^{2}$ \\
\hline Mass of the ball & $m$ & $0.022 \mathrm{Kg}$ \\
\hline PMDC motor rotor mass & $m_{R}$ & $0.050 \mathrm{Kg}$ \\
\hline Servo arm mass & $m_{s}$ & $0.010 \mathrm{~kg}$ \\
\hline Link arm mass & $m_{L}$ & $0.010 \mathrm{~kg}$ \\
\hline Beam mass & $m_{B}$ & $0.025 \mathrm{~kg}$ \\
\hline Radius of PMDC motor rotor & $r$ & $0.02 \mathrm{~m}$ \\
\hline Length of link arm & $L$ & $0.375 \mathrm{~m}$ \\
\hline Width of the servo Arm & $l$ & $0.010 \mathrm{~m}$ \\
\hline Length of servo arm & $a$ & $0.1875 \mathrm{~m}$ \\
\hline Half beam length & $b$ & $0.375 \mathrm{~m}$ \\
\hline Beam width & $p$ & $0.025 \mathrm{~m}$ \\
\hline Rolling friction constant for ball & $B$ & $0.018 \mathrm{~N} . \mathrm{m} / \mathrm{rad} / \mathrm{sec}$ \\
\hline Rotational friction constant PMDC motor rotor & $B_{R}$ & $0.08 \mathrm{~N} \cdot \mathrm{m} / \mathrm{rad} / \mathrm{sec}$ \\
\hline Rotational friction constant for beam about $\mathrm{O}_{2}$ & $B_{2}$ & $0.1 \mathrm{~N} . \mathrm{m} / \mathrm{rad} / \mathrm{sec}$ \\
\hline Torque constant for PMDC motor & $k_{\tau}$ & 0.008 N.m/Amp \\
\hline Back EMF constant for PMDC motor & $k_{m}$ & $0.015 \mathrm{~V} / \mathrm{rad} / \mathrm{sec}$ \\
\hline Ball position sensing linear potentiometer constant & $k_{D}$ & $5 \mathrm{~V} / \mathrm{m}$ \\
\hline Beam angle sensing accelerometer constant & $k_{\theta}$ & $4 \mathrm{~V} / \mathrm{rad}$ \\
\hline Beam angular velocity gyroscopic constant & $k_{d \theta / d t}$ & $2.5 \mathrm{~V} / \mathrm{rad} / \mathrm{sec}$ \\
\hline Servo arm angular potentiometer constant & $k_{\phi}$ & $3.5 \mathrm{~V} / \mathrm{rad}$ \\
\hline Armature current hall effect constant & $k_{i}$ & $4.5 \mathrm{~V} / \mathrm{A}$ \\
\hline Moment of inertia for rotor-servo-link arm assembly & $J_{1}$ & $5.863 \mathrm{e}-3 \mathrm{Kg} \cdot \mathrm{m}^{2}$ \\
\hline Moment of inertia for servo arm & $J_{s}$ & 4.68e-4 Kg.m² \\
\hline Moment of inertia for link arm & $J_{L}$ & $1.8721-3 \mathrm{Kg} \cdot \mathrm{m}^{2}$ \\
\hline Moment of inertia for motor rotor & $J_{R}$ & $3.52 \mathrm{e}-3 \mathrm{Kg} \cdot \mathrm{m}^{2}$ \\
\hline Moment of inertia for beam & $J_{2}$ & 2.93e-7 Kg.m $\mathrm{m}^{2}$ \\
\hline Armature resistance & $R_{a}$ & $0.8 \mathrm{Ohm}$ \\
\hline Armature inductance & $L_{a}$ & $0.245 \mathrm{H}$ \\
\hline
\end{tabular}

